\title{
DISCLAIMER
}

\begin{abstract}
This report was prepared as an account of work sponsored by an agency of the United States Government. Neither the United States Government nor any agency thereof, nor any of their employees, makes any warranty, express or implied, or assumes any legal liability or responsibility for the accuracy, completeness, or usefulness of any information, apparatus, product, or process disclosed, or represents that its use would not infringe privately owned rights. Reference herein to any specific commercial product, process, or service by trade name, trademark, manufacturer, or otherwise does not necessarily constitute or imply its endorsement, recommendation, or favoring by the United States Government or any agency thereof. The views and opinions of authors expressed herein in not necessarily state or reflect those of the United States Government or any agency thereof.
\end{abstract}

By acceptance of this articie, the publisher recognizes that the U.S Government retains a nonexclusive, royalty-free license to publish or reproduce the published form of inis contribution. or to allow others to do so, for U.S Government purposes 


\section{Antimatter Gravity Experiment}

RONALD E. BROWN, J.B. CAMP, and T.W. DARLING

Los Alamos National Laboratory, Los Alamos, New Mexico 87545, USA

An experiment is being developed to measure the acceleration of the antiproton in the gravitational field of the earth. Antiprotons of a few $\mathrm{MeV}$ from the LEAR facility at CERN will be slowed, captured, cooled to a temperature of about $10 \mathrm{~K}$, and subsequently launched a few at a time into a drift tube where the effect of gravity on their motion will be determined by a time-of-flight method. Development of the experiment is proceeding at Los Alamos using normal matter. The fabrication of a drift tube that will produce a region of space in which gravity is the dominant force on moving ions is of major difficulty. This involves a study of methods of minimizing the electric fields produced by spatially varying work functions on conducting surfaces. Progress in a number of areas is described, with stress on the drift-tube development.

\section{Introduction}

We are developing an experiment to measure the acceleration of the antiproton in the gravitational field of the earth. No measurement has ever been made of the gravitational interactions of antimatter. Supersymmetric theories that attempt to unify quantum mechanics did gravity generate graviscala0r and gravivector partners of the graviton, thereby yielding possible composition dependences and deviations from the inverse square law [1]. Searches for such effects in the matter-matter interaction have been made over a variety of distance scales [2,3], thereby placing limits on the strengths and ranges of such interactions. An antimatter-matter measurement is of special interest because the gravivector term would change sign from that of a matter-matter experiment, implying that an antiproton might fall faster toward the earth than a proton does [1]. Independent of such ideas, however, a measurement of the gravitational acceleration of the antiproton will be a direct test of whether or not the antiproton obeys the weak equivalence principle. 


\section{Gravity experiment}

Antiprotons of a few $\mathrm{MeV}$ from the LEAR facility at CERN will be slowed to tens of $\mathrm{keV}$ by an energy degrading foil, caught in a large electromagnetic trap (the catching trap), and cooled to tens of eV. These antiprotons will then be transferred to a much smaller trap (the launching trap), where they will be cooled to a temperature of about $10 \mathrm{~K}$ and subsequently launched a few at a time into a vertical, shielding, metallic drift tube of length $L \approx 1 \mathrm{~m}$, where the effect of gravity on their motion will be determined by a time-of-flight method. This method involves measuring the distribution of flight times through the drift tube, thereby determining a cutoff time $t_{c}=(2 \mathrm{~L} / \mathrm{g})^{1 / 2}$ (about $0.4 \mathrm{sec}$ ) from which $\mathrm{g}$ can be extracted. Measurements using antiprotons will be compared with measurements using $\mathrm{H}^{-}$ions in order to eliminate some systematic errors. Development of the experiment is proceeding at Los Alamos using normal matter. See refs. [4,5] for more discussion of the experimental plan.

Care must be taken to control vertical forces on the drifting ions. A vertical electric field of $10^{-7} \mathrm{~V} / \mathrm{m}$ or a magnetic inhomogeneity $\triangle B / B$ of $10^{-4}$ over one meter will give a force on the antiproton equal to that given by the earth's gravity. In the pioneering electron experiment of Witteborn and Fairbank [6], it was believed that stray electric fields could be lowered to about $10^{-11} \mathrm{~V} / \mathrm{m}$, although there is still controversy over whether or not such low fields were actually attained. The antiproton experiment has advantages over the electron experiment in that the larger antiproton mass allows a measurement with a considerably higher background electric field, and a crlibration ion $\mathrm{H}^{-}$exists.

The fabrication of a drift tube that will allow ions to experience gravity as the dominant force is of major difficulty. This involves a study of the electric fields produced by spatially varying work functions on conducting surfaces (the patch effect). Drift-tube prototypes with a variety of surface coatings will be tested (section 6). Initial screening of the coatings is being carried out with a Kelvin probe (section 5 ).

\section{Trapping studies}

The earlier work [4] on the trapping of beam particles in electromagnetic 
traps has continued [5]. At LEAR, we have used foils to degrade 5-MeV antiprotons to tens of $\mathrm{keV}$ and have verified the expected yield as a function of emerging antiproton energy. At the Los Alamos Ion beam Facility, the energy of a $5-\mathrm{MeV}$ proton beam has been degraded by metallic foils to tens of $\mathrm{keV}$ and trapped in a room-temperature, $50-\mathrm{cm}$ long, cylindrical trap situated in a $6-\mathrm{T}$ magnetic field. The trapping voltage has been in the $5-$ to $15-\mathrm{kV}$ range, but we are attempting to raise this into the $30-$ to $50-\mathrm{kV}$ range. To this end we will convert the trap to run at cryogenic temperatures to reduce the pressure below $10^{-10}$ torr. This should help alleviate glow discharges that can develop as the trap voltage is raised. We also plan to load electrons into this trap to study their cyclotron cooling characteristics with a view toward using electron cooling of antiprotons at LEAR. It is reassuring to know that G. Gabrielse and collaborators $[7,8]$ have already used foil degrading to trap and cool some tens of thousands of antiprotons in a $3-\mathrm{kV}$ trap at LEAR.

Studies of problems associated with launching traps and resistive cooling techniques are being investigated at Texas A\&M [9] and Los Alamos. The goal is to guarantee that a sufficient number of slow particles can be launched into the drift tube.

\section{Drift-tube surface}

The surface of even the best of conductors is not at a constant electrostatic potential because it consists of many crystal faces and/or chemically different regions (patches) that can have differing work functions $[10,11]$. This work function variation gives a spatially nonuniform surface potential that produces an electric field exterior to the surface of the conductor. The rms field along the axis of our uncoated drift tube produced by a typical random patch distribution on its inner surface is larger than the gravitational equivalent. However, we plan to coat that surface with a suitable material to reduce this field significantly. Such materials are being studied with the Kelvin probe apparatus described in section 5. Elimination of this patch effect to the appropriate level is the most difficult of the technical challenges in developing a drift tube.

In ref. [6] an estimate of the rms deviation in potential $\Phi_{\mathrm{rms}}$ seen by an ion traversing the axis of a cylinder of radius $a$ is given as 
$\Phi_{\mathrm{rms}}=0.6 \Delta \phi \lambda / \alpha$

where $\Delta \phi$ is the rms change in work function on the surface, and $\lambda$ is a characteristic patch dimension. Other derivations [12-14] yield results similar to eq. (1), having only minor differences in the numerical constant. Eq. (1) and the relations of refs. [12-14] are basically given by the statistical quantity $\Delta \phi / \sqrt{ } \mathrm{N}_{\mathrm{e}}$, where $\mathrm{N}_{\mathrm{e}}$ is the effective number of patches viewed by the ion, i.e. $\mathrm{N}_{\mathrm{e}}=$ (effective surface area viewed by the ion)/(area of a typical patch). For a cylindrical surface viewed by an ion on the axis, $\mathrm{N}_{\mathrm{e}}$ is approximately $(2 \pi a) a / \lambda^{2}$, which yields the form of eq. (1). Taking the reasonable values $\Delta \phi=0.1 \mathrm{~V}, \lambda=$ $1 \mu \mathrm{m}$, and $a=1 \mathrm{~cm}$, we find $\Phi_{\mathrm{rms}}=6 \mu \mathrm{V}$. This value would not allow gravity measurements for protons or antiprotons, which would require $\Phi_{\mathrm{rms}}<0.1 \mu \mathrm{V}$ over a 1-m drift length (section 2). However, it would allow a gravity measurement on a heavier ion, such as Xe, which "only" requires $\Phi_{\mathrm{rms}}<13 \mu \mathrm{V}$.

\section{Kelvin-probe experiments}

In order to obtain information on work-function uniformity for materials that might be used for coating the drift tube, we have developed a vibrating electrometer apparatus (Kelvin probe) to study small samples of surfaces under a variety of conditions. In these experiments we do not attempt to measure absolute work functions, but only their spatial variations.

A hollow, gold-plated, $0.8-\mathrm{mm}$ diameter, aluminum probe is riveted to a molybdenum reed, which in turn is affixed to a ceramic, piezoelectric bimorph. The probe protrudes $0.25 \mathrm{~mm}$ through a stainless steel ground plane. That system is mounted on $\mathrm{an}$. xy table to scan the surfaces of interest. The surfaces to be studied are coated onto a bronze substrate of dimensions $2.4 \mathrm{~cm} \times$ $1.6 \mathrm{~cm} \times 4.8 \mathrm{~mm}$. Bronze is the material used for the drift-tube body (section 6). The scanning apparatus and sample are positioned inside an ultra-high vacuum enclosure, and the sample can be heated with a lamp from outside of the enclosure.

With the probe positioned $\sim 0.5 \mathrm{~mm}$ above the sample and vibrating with an amplitude of $\sim 0.2 \mathrm{~mm}$ and a frequency of $\sim 500 \mathrm{~Hz}$, a current is induced in the sample through the time variation of the probe-to-surface capacitance. This 
current is sent through a vacuum feedthrough to a high-gain amplifier and then into a lock-in amplifier referenced to the probe frequency. The lock-in output is integrated and fed back to the probe as a bias voltage. The system then adjusts itself until the probe potential is equal to the local potential at the sample surface. The probe bias voltage $V_{\mathrm{s}}$ necessary to accomplish this is a measure of the local work function of the sample. $V_{\mathbf{S}}$ is then measured at different positions on the sample to determine the spatial uniformity of its work function. The spatial resolution of the Kelvin probe is $1.5 \mathrm{~mm}$, and its sensitivity is $1 \mathrm{mV}$.

We have investigated surfaces of varying degree of conductivity, crystal linity, and reactivity (Table 1 ). The measurement procedure begins with a 1-day bake of the apparatus followed by a 5-hr: cooldown. The resultant pressure was less than $7 \times 10^{-10}$ torr for all measurements except that of polyanilene, where a lower bakeout temperature limited the final vacuum to $6 \times 10^{-}$ 9 torr. The surface was then scanned. Next, the surface was heated with the heat lamp for $12 \mathrm{hr}$, after which the surface was rescanned. A nickel coating was used as a diffusion barrier for $\mathrm{Al}$ and $\mathrm{Au}$. Table 1 lists the rms variations $\sigma$ in surface potential following the vacuum bake $\left(\sigma_{1}\right)$ and surface heating $\left(\sigma_{2}\right)$. $\mathrm{T}$ is the equilibrium surface temperature reached during baking or heating. Gold and graphite displayed no surface potential structure at the limit of sensitivity of the Kelvin probe. It appears, therefore, that it is necessary to use nonreactive surfaces for the drift tube to keep adsorption effects to a minimum.

Converting the measurements $\sigma$ to $\Phi_{\mathrm{rms}}$ for an ion moving on the axis of a drift tube of radius $a$ requires multiplying $\sigma$ by the ratio of the square root of the surface area viewed by the probe to that viewed by the ion. Thus we have $\Phi_{\mathrm{rms}}$ $\approx \sigma \sqrt{ }\left(\pi r^{2} / 2 \pi c^{2}\right) \approx \sigma / 20$, where $r$ is the effective probe radius of $0.75 \mathrm{~mm}$ and $a$ is $1 \mathrm{~cm}$. Taking $\sigma<1 \mathrm{mV}$ for graphite or gold, we obtain $\Phi_{\mathrm{rms}}<50 \mu \mathrm{V}$. The actual $\Phi_{\mathrm{rms}}$ may be considerably smaller chan this upper limit, with a lower limit perhaps established by the size of the gold grains or graphite flakes. Finding the actual $\sigma$ for materials that are at the sensitivity limit of $1 \mathrm{mV}$ will require use of the test apparatus described in section 6. A further description of the Kelvin-probe work is given in ref. [15]. 


\section{Drift-tube testing}

A critical component in the experiment is a drift tube that can provide a region of space where the forces on the moving ions will be dominated by gravity. There are many effects that can cause spurious results in a gravity experiment using a drift tube [6]. The patch effect is the most difficult problem to resolve.

With the drift-tube test apparatus we will study the motion of several ion species through drift tubes under varying experimental conditions. This apparatus consists of four modules: (1) Ion Source, (2) Drift Tube, (3) Solenoid Magnet, and (4) Detector. Items (2)-(4) will be operated in a cryogenic environment. Measurements of time-of-flight spectra for the passage of ions through the drift tube as various parameters are changed will allow deductions to be made about field conditions in the drift region. In particular, the observation of slowly moving particles will indicate low ambient fields in the drift region.

The four modules contribute to the test experiment as follows:

(1) The pulsed ion source will be mounted above the drift tube outside of the cryogenic environment. It will be capable of furnishing ions of differing masses and charge states. We plan to start with Xe ions, whose gravityequivalent electric field is only $10^{-5} \mathrm{~V} / \mathrm{m}$. After observing the effect of gravity on $\mathrm{Xe}$, we will work our way down in mass to protons. We have ordered a commercial electron-cyclotron-resonance source [16] because of its high ionization efficiency and reasonably small energy spread. Until the ion source arrives, we are testing the remainder of the system using electrons produced by a field emitter.

(2) The test drift tubes are accurately machined from bronze, are $50-\mathrm{cm}$ long, have an internal diameter of $2.54 \mathrm{~cm}$, and will be internally coated with different types of material to study the patch effect. In its cryogenic environment, the temperature of the tube can be varied from 4 to $20 \mathrm{~K}$. External electric fields are well shielded from the tube interior, allowing a "field-free" drift region about $30 \mathrm{~cm}$ long. This effective drift length $L$ can be varied by changing the potentials on electrodes at either end of the drift tube. Known, small electric fields can be imposed on the drift region by running electric currents along the tube. Bronze was chosen for the drift-tube body to maintain a reasonably high electric resistance at low temperatures in generate these 
imposed fields.

(3) To keep slow ions from being deflected by image forces, a guiding, axial magnetic field is used. High field uniformity is necessary in order to avoid magnetic gradient forces on the ions. Computer simulations show that $\triangle B / B$ should be $10^{-5}$ or lower. We have purchased [17] a superconducting solenoid magnet of homogeneity better than $10 \mathrm{ppm}$ over a field range of 0.5 to 2.0 tesla and over a cylindrical volume $50 \mathrm{~cm}$ long by $1 \mathrm{~cm}$ diameter.

(4) A particle detector is situated below the drift tube. The microchannel plate electron multiplier is at present the detector of choice. It is bakable and compatible with the ultra high vacuum. It gives a fast pulse for time-of-flight measurements, is relatively insensitive to axial magnetic fields, and is expected to operate at least down to liquid-nitrogen temperature. We will soon be studying the characteristics of these devices at low temperatures. The temperature of the detector can be kept above its $4 \mathrm{~K}$ environment by a heater.

At present we are operating a room temperature test system at a pressure of 1 to $2 \times 10^{-10}$ torr. The system is surrounded by a $30-\mathrm{G}$ solenoid magnet and contains an electron field emitter, various electrodes, an uncoated drift tube, and a microchannel plate. We are studying the optics and flight time for the electrons moving from the emitter to the detector. In the time-of-flight spectrum we have observed late arriving electrons from a trap somewhere in the system. We are pursuing this question vigorously, as it is absolutely essential to be able to distinguish between slow particles and late ones coming from traps.

\section{Conclusion}

Development of an experiment is underway to measure the acceleration of the antiproton in the earth's gravitational field. No measurement of the gravitational interactions involving antimatter has ever been performed. The major problems being addressed are: trapping of ions, sufficient cooling of the trapped ions, launching of ions into a drift tube, and reduction of the patch effect in the drift tube to the gravitational level. 
The technical expertise that D.C. Lizon, R. Martinez, and C.B. Webb have so diligently applied to the antiproton gravity experiment is gratefully acknowledged. We are indebted to the other members of the experimental collaboration for work presented here. Current members include: M.H. Holzscheiter, P. Dyer D.B. Holtkamp, N. Jarmie, N.S.P. King, M.M. Schauer (Los Alamos National Laboratory); F. C. Witteborn (NASA/Ames Research Center); S. Cornford, R.A. Kenefick, K. Hosea (Texas A \& M. Univ.); M. Mizdor, R. Ristinen (Univ. of Colorado). Many members of the scientific community have supported this experiment through help, discussions, and interest. It is impossible to adequately acknowledge them all here. This work was supported by the U.S. Department of Energy under Contract No. W7405 ENG-36.

\section{References}

[1] T. Goldman, R.J. Hughes, and M.M. Nieto, Phys. Lett. 171B (1986) 217; Scientific American 258, no. 3 (1988) 48; Australian Physicist 25 (1988) 259; M.M. Nieto and T. Goldman, submitted to Phys. Reports (1990).

[2] B.R. Heckel, E.G. Adelberger, C.W. Stubbs, Y. Su, H. E. Swanson, G. Smith, and W.F. Rogers, Phys. Rev. Lett. 63 (1989) 2705, and references therein.

[3] F.D. Stacey, G.J. Tuck, G.I. Moore, S. C. Holding, B.D. Goodwin and R. Zhou, Rev. Mod. Phys. 59 (1987) 157; M.E. Ander et al., Phys. Rev. Lett. 62 (1989) 985; M.M. Nieto et al.,Phys. Lett. B 228 (1989) 448.

[4] R.E. Brown, in: Proc. 2nd Conf. on the Intersections between Particle and Nuclear Physics, Lake Louise, Canada (May, 1986) ed. D.F. Geesamen, AIP publ. 150 (American Institute of Physics, New York, 1986) p 436.N. Jarmie, Nucl. Instr. and Meth. B24/25 (1987) 437; M.H. Holzscheiter, R.E. Brown, N. Jarmie, and D.C. Lizon, Phys. Scripta T22 (1988) 290; P. Dyer, J. Camp, M.H. Holzscheiter, and S. Graessle, Nucl. Instr. and Meth. B40/41 (1989) 485; M.H. Holzscheiter et al., Atomic Physics Conf., Michigan, to be published (1990).

[5] M.H. Holzscheiter, Stockholm Conf., to be published (1990).

[6] F.C. Witteborn and W.M. Fairbank, Phys. Rev. Lett. 19 (1967) 1049 ; Rev. Sci. Instr. 48 (1977) 1 ; F.C. Witteborn, Ph. D. thesis, Stanford University (1965). 
[7] G. Gabrielse, X. Fei, L. Haarsma, S.L. Rolston, R. Tjoelker, T.A. Trainor, H. Kalinowsky, J. Haas, and W. Kells, Phys. Scripta T22 (1988) 36.

[8] G. Gabrielse, X. Fei, L.A. Orozco, R.L. Tjoelker, J. Haas, H. Kalinowsky, T.A. Trainor, and W. Kells, Phys. Rev. Lett. 63 (1989) 1360; 65 (1990) 1317.

[9] R.A. Kenefick, private communication.

[10] C. Herring and M.H. Nichols, Rev. Mod. Phys. 21 (1949) 185.

[11] T.W. Darling, Ph.D. Thesis, University of Melbourne, Australia (1989),

[12] M.S. Rzchowski and J.R. Henderson, Phys. Rev. A 38 (1988) 4622.

[13] T.W. Darling, F. Rossi, G.I. Opat, and G.F. Moorhead, to be published.

[14] G.I. Opat, G.F. Moorhead, and F. Rossi, to be published.

[15]. J.B. Camp, T.W. Darling, and R.E. Brown, submitted to J. Appl. Phys.

[16] Kimball Physics Inc., Wilton NH.

[17] Oxford Instruments Ltd., Eynsham, England. 
Table 1

Measured rms variations $\sigma$ in surface-potentials.

The sensitivity limit for $\sigma$ is $1 \mathrm{~m} \mathrm{~V}$.

\begin{tabular}{lrrrr}
\hline Surface & $\begin{array}{c}\mathrm{T} \text { (bake) } \\
\left({ }^{\circ} \mathrm{C}\right)\end{array}$ & $\begin{array}{c}\sigma_{1} \\
(\mathrm{mV})\end{array}$ & $\begin{array}{c}\mathrm{T} \text { (heat) } \\
\left({ }^{\circ} \mathrm{C}\right)\end{array}$ & $\begin{array}{c}\sigma_{2} \\
(\mathrm{mV})\end{array}$ \\
\hline Bronze $^{\mathrm{a})}$ & 110 & 13 & 275 & 24 \\
NiP b) & 60 & 12 & 75 & 18 \\
Polyaniline c) & 40 & 40 & 50 & 28 \\
Al & 110 & 2 & 150 & 9 \\
Cu & 110 & 3 & 175 & 6 \\
Au & 110 & 1 & 130 & 1 \\
Graphite d) & 110 & 1 & 350 & 1 \\
\hline
\end{tabular}

a) Grade $A$, used for drift-tube body.

b) Amorphous.

c) Conductive polymer.

d) Aerosol spray, 1- $\mu \mathrm{m}$ flakes, Acheson Colloids Co. 

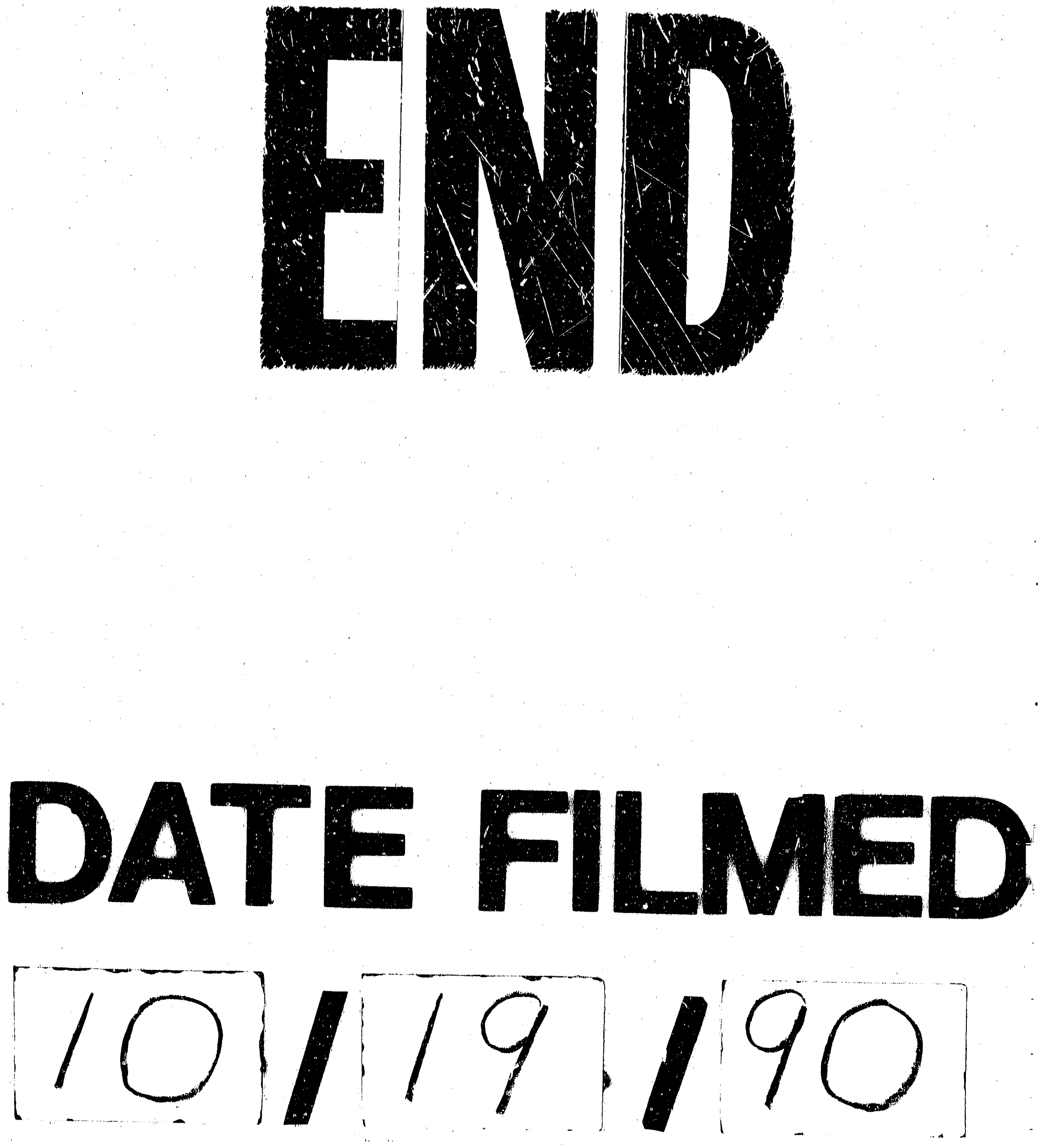